\title{
Acidente com material biológico no atendimento pré-hospitalar móvel: realidade para trabalhadores da saúde e não saúde
}

\author{
Accident with biological material at the prehospital mobile care: reality for health and non-healthcare workers \\ Accidente con material biológico en la atención pre hospitalario móvil: realidad para trabajadores de \\ salud y no-salud
}

\author{
Anaclara Ferreira Veiga Tipple', Elisangelo Aparecido Costa Silva", Sheila Araújo Teles', \\ Katiane Martins Mendonça"I, Adenícia Custódia Silva e Souza", Dulcelene Sousa Melo"'I \\ ' Universidade Federal de Goiás, Faculdade de Enfermagem, Programa de Pós-Graduação em Enfermagem. Goiânia-GO, Brasil. \\ "Pontifícia Universidade Católica de Goiás, Departamento de Enfermagem, Nutrição e Fisioterapia, \\ Curso de Pós-Graduação em Enfermagem. Goiânia-GO, Brasil. \\ I'" Universidade Federal de Goiás, Faculdade de Enfermagem, \\ Programa de Pós-Graduação em Enfermagem (Doutoranda). Goiânia-GO, Brasil.
}

Submissão: 20-03-2012 Aprovação: 21-05-2013

RESUMO

Estudo analítico transversal, com objetivos de identificar a prevalência e caracterizar os acidentes com material biológico entre profissionais do Atendimento Pré-Hospitalar (APH) e comparar os comportamentos de risco adotados entre os grupos saúde e não saúde que podem influenciar na ocorrência e na gravidade destes acidentes. Dados foram obtidos por meio de questionário aplicado a todos os trabalhadores do APH de Goiânia - GO. Verificou-se alta prevalência de acidentes envolvendo material biológico que, apesar de maior no grupo saúde, também acometeu o grupo não saúde. Comportamentos de risco, estatisticamente significativos ( $p<0,05)$, para ocorrência de acidentes em ambos os grupos: não uso de luvas, máscara e óculos de proteção, descarte inapropriado de perfuro-cortante, vestimenta incompleta, reencape de agulhas e não imunização contra hepatite B. Os dados evidenciaram a importância da adesão às medidas preventivas por ambos os grupos e a necessidade de estruturação e implementação de um sistema de vigilância e controle deste tipo de acidente.

Descritores: Exposição a Agentes Biológicos; Assistência Pré-hospitalar; Exposição Ocupacional.

\section{ABSTRACT}

Analytical transversal study that was conducted with the objectives of identifying the prevalence and characterizing the accidents with biological material among professionals in pre-hospital service (PHS) and comparing the risk behaviors adopted by healthcare and non-healthcare groups that can affect the occurrence and seriousness of such accidents. Data were obtained by questionnaire applied to all PHS workers in Goiânia-GO. The study revealed a high prevalence of accidents involving biological material which, although higher for the healthcare group, also affected the non-healthcare group. There were significant ( $p$ $<0.05$ ) risk behaviors for these accidents in both groups: not using gloves, masks or eye protectors; inappropriate disposal of sharps; inadequate dress; re-capping of needles; and a lack of immunization against hepatitis B. The results underscore the importance of both groups in adhering to preventive measures, and further point to the need to structure and implement vigilance and control system for this type of accident.

Key words: Exposure to Biological Agents; Prehospital Care; Occupational Exposure.

\section{RESUMEN}

Estudio transversal analítico con objetivos de identificar y caracterizar la prevalencia de accidentes con material biológico entre profesionales de la atención pre hospitalaria (APS) y comparar los comportamientos de riesgo adoptados entre los grupos salud y no-salud que pueden influir en la aparición y gravedad de estos accidentes. Los datos fueron recolectados con cuestionario aplicado a todos los trabajadores de la APS de Goiânia - GO. Hubo alta prevalencia de accidentes con material biológico que, aunque mayor en el grupo salud, también ocurrió en no-salud. Las conductas de riesgo, estadísticamente significativas ( $p<0,05)$, para accidentes en ambos los grupos: sin uso de guantes, máscara y gafas de protección, descarte inadecuado de objetos punzocortantes, ropa incompleta, tapar agujas y no vacunación para hepatitis B. Los datos mostraron la importancia de ambos grupos adherir a las medidas preventivas y la necesidad de estructurar e implementar un sistema para monitorear y controlar este tipo de accidentes.

Palabras clave: Exposición a Agentes Biológicos; Atención Pre hospitalaria; Exposición Profesional.

\section{AUTOR CORRESPONDENTE Anaclara Ferreira Veiga Tipple E-mail: anaclara@fen.ufg.br}




\section{INTRODUÇÃO}

O serviço de APH na cidade de Goiânia-GO, localizada na região Centro-Oeste do Brasil, assim como no restante do país, tem um histórico vinculado à instituição militar. Em Goiânia, a partir de 2000 o Corpo de Bombeiros Militar do Estado de Goiás (CBMGO) foi estruturado para o Atendimento Pré-Hospitalar (APH), por meio do Grupo de Resgate Pré-hospitalar (GRPH), e no mesmo ano, houve a implantação de outro modelo de APH, o Sistema Integrado de Atendimento ao Trauma e Emergência (SIATE).

Em 2004, o Serviço de Atendimento Móvel de Urgência (SAMU), também integrante do APH no país, começou a atuar no município de Goiânia-GO e em 2011, Goiás faz parte dos dois únicos Estados que possuem 100\% de seus territórios atendidos pelo SAMU, no Brasil, além de Sergipe ${ }^{(1)}$.

Atualmente existem dois serviços de $\mathrm{APH}$, públicos, no município de Goiânia-GO: o SIATE (órgão da Secretaria Estadual de Saúde que atua em convênio com o Grupamento de Salvamento e Emergência (GSE) do CBMGO) e o SAMU, na esfera municipal.

A equipe do APH inclui profissionais oriundos da área da saúde: médicos, enfermeiros, auxiliares e técnicos de enfermagem; e não oriundos da área da saúde: telefonista, rádio-operador, condutor de veículos de urgência, bombeiros militares, profissionais responsáveis pela segurança (como policiais militares e rodoviários) e outros ${ }^{(2)}$.

No caso de bombeiros militares, especificamente, a legislação brasileira permite a realização de suporte básico de vida, com ações, consideradas não invasivas, sob supervisão médica direta ou à distância, obedecendo aos padrões de capacitação e atuação previstos em normativas nacionais(2).

Os trabalhadores dos serviços de APH realizam atendimentos de urgência, que podem ser caracterizados como diretos (por profissionais oriundos da área da saúde) e indiretos (por profissionais não oriundos da área da saúde). Rotineiramente, esse trabalho envolve procedimentos que são realizados sob condições de estresse, com alto risco de morte das vítimas, em condições frágeis de estrutura física, o que, particularmente, torna os profissionais do APH mais susceptíveis a acidentes envolvendo material biológico $(\mathrm{MB})^{(3)}$. Estudos apresentam taxas elevadas de acidentes com MB entre esses trabalhadores $^{(4-10)}$.

Embora os profissionais oriundos da área da saúde apresentem maior risco de acidentes envolvendo $M B$, haja vista a natureza dos procedimentos que executam, os demais também participam de situações que envolvem o risco biológico (RB), entretanto, a discussão da comunidade científica predomina o enfoque aos primeiros.

Diante disso, e por considerar que as urgências não se constituem especialidade médica ou de enfermagem e ainda, por julgar que atenção a esta área ainda é insuficiente nos cursos de graduação, o Ministério da Saúde implantou os Núcleos de Educação em Urgências com a finalidade de qualificar os profissionais (oriundos e não da área de saúde) para atuarem em Serviços de APH Móvel(2). Essa qualificação inclui noções de medidas de prevenção e controle de infecção para todos os profissionais da área da saúde e para aqueles não oriundos desta área, limita-se aos profissionais da área de segurança, bombeiros e condutores de veículos de urgência (tipo B, C e D).

Sabe-se que as medidas de Precaução Padrão (PP) $)^{(11)}$ tem como essência considerar o risco como universal. De acordo com essas medidas, qualquer indivíduo é um potencial portador de microrganismos infecciosos e, portanto, medidas preventivas, como a adoção de equipamentos de proteção e a prática de higienização das mãos, devem ser adotadas, independente do diagnóstico, conhecido ou presumível, do paciente.

Nos serviços de APH onde o risco de acidentes com MB é potencializado pelas peculiaridades que envolvem cada atendimento as medidas de PP deveriam ser incorporadas por todos os profissionais e, portanto, integrar a pauta de educação permanente deste grupo.

Diante dos escassos estudos que abordam o RB da equipe de profissionais, oriundos e não oriundos da área da saúde do APH móvel e a inexistência de dados relacionados com esta população na região Centro-Oeste é que este estudo faz-se necessário.

Identificar a prevalência e caracterizar os acidentes com material biológico entre os trabalhadores do Atendimento Pré-hospitalar em Goiânia, Goiás e comparar os comportamentos de risco adotados entre os grupos saúde e não saúde que podem influenciar na ocorrência e na gravidade destes acidentes.

\section{MÉTODOS}

Estudo transversal, analítico realizado nos serviços públicos de atendimento de urgência e emergência pré-hospitalar do município de Goiânia-GO. Esses serviços são formados pelo SIATE e SAMU.

A população foi constituída por todos os profissionais que atuavam nos serviços do SIATE e SAMU de Goiânia-GO. Foram incluídos médicos, enfermeiros, técnicos em enfermagem, condutores e socorristas que participavam de forma ativa no atendimento de urgência e emergência. Foram excluídos, os profissionais que desempenhavam serviços administrativos e aqueles em férias ou licença médica, no período da coleta de dados.

Considerou-se como variável de desfecho a ocorrência de acidente com MB durante o tempo de trabalho nesses serviços e variáveis de predição sexo, idade, categoria profissional, tempo de atuação no APH, carga horária semanal, tempo de experiência e adesão a algumas medidas de biossegurança como: uso de equipamentos de proteção (luvas, máscara e óculos protetores), descarte de perfurocortantes em local adequado, não reencape de agulhas, uso do uniforme completo (com as mangas até o punho) e imunização para hepatite B e tétano.

Os dados foram obtidos mediante aplicação de um questionário estruturado, auto-aplicável, constituído por questões objetivas. O instrumento foi avaliado por três especialistas em controle de infecção e submetido a teste piloto em um serviço de APH de outro município. 
A coleta dos dados ocorreu no período de julho a agosto de 2006, no momento da troca de plantão dos profissionais. Os instrumentos eram devolvidos ao pesquisador logo após a sua aplicação.

Para o processamento e análise dos dados, utilizou-se o programa Epi-Info, versão 3.3 e o pacote estatístico SPSS/IBM, versão 10.0 for Windows. Prevalências foram calculadas com intervalo de confiança de 95\%. Realizou-se a análise univariada e multivariada $\mathrm{O}$ teste de qui-quadrado e exato de Fisher foram utilizados para testar a significância de diferenças entre as proporções. Valores de $p<0,05$ foram considerados estatisticamente significativos.

Para fins de análise, os trabalhadores foram agrupados em profissionais dos grupos saúde (enfermeiros, médicos e técnicos em enfermagem) e não saúde (condutores e socorristas - bombeiros militares).

O estudo foi aprovado por um Comitê de Ética em Pesquisa (protocolo de aprovação número 001-006). Os sujeitos foram informados sobre objetivos e aspecto voluntário da participação, com preservação do anonimato e direito de se retirarem do estudo, em qualquer momento, sem sofrerem prejuízos. No caso de aceite, o profissional assinou o Termo de Consentimento Livre e Esclarecido.

\section{RESULTADOS}

O total de trabalhadores elegíveis para o estudo era de 202, nove estavam em férias, quatro em licença médica e 12 recusaram. Participaram 177 indivíduos representando 87,6\% da população pretendida. Dentre eles, 101 integravam o grupo saúde e 76 o não saúde. A Tabela 1 apresenta a caracterização dos sujeitos.

As variáveis que apresentaram diferenças estatísticas com as demais foram o sexo masculino e o tempo de atuação superior a cinco anos $(p<0,05)$.

A prevalência global desses acidentes foi de 41,2\% (73/177) entre os profissionais do APH. Considerando os grupos saúde e não saúde, a prevalência de acidentes foi de 58,4\% (59/101) e $18,4 \%$ (14/76), respectivamente.

Dentre os 73 acidentes, a maioria envolveu profissionais enfermeiros $(28,7 \% ; 21 / 44)$ seguidos por médicos $(26,1 \%$; $19 / 26)$, técnicos em enfermagem7 $(26,1 \% ; 19 / 31)$, socorristas $(15,0 \% ; 11 / 34)$ e condutores $(4,1 \% ; 3 / 42)$. Os acidentes foram caracterizados em graves $(45 / 73 ; 61,7 \%)$ e não graves $(28 / 73 ; 38,3 \%)$, conforme as características da exposição ao $\mathrm{MB}$.

Os acidentes graves foram: ferimentos com material previamente inserido em acesso vascular $(15 ; 20,5 \%)$, ferimento profundo com material contaminado com sangue (13; $17,9 \%)$, exposição a grande quantidade de sangue $(07 ; 9,6 \%)$, exposição a $\mathrm{MB}$ em pele ou mucosa não íntegra do profissional $(05 ; 6,8 \%)$, tamanho da área de exposição ao MB (03; $4,1 \%)$, elevado tempo de exposição ao $M B(01 ; 1,4 \%)$ e envolvimento de $\mathrm{MB}$ proveniente de paciente portador do Vírus da Imunodeficiência Humana fora de tratamento $(01 ; 1,4 \%)$.

Os acidentes considerados não graves foram: contatos rápidos com MB em pele íntegra $(24 ; 32,9 \%)$, ferimentos superficiais com agulha de sutura $(02 ; 2,7 \%)$ e arranhões superficiais com material perfurocortante $(02 ; 2,7 \%)$.

A maioria dos acidentes envolveu sangue $(63 ; 86,3 \%)$, saliva $(06 ; 8,3 \%)$, líquido pleural $(02 ; 2,7 \%)$ e secreção gástrica $(02 ; 2,7 \%)$.

O envolvimento com perfurocortantes ocasionou 32 $(43,8 \%)$ acidentes, sendo: ferimento com material previamente utilizado em acesso vascular $(15 ; 20,5 \%)$, ferimento profundo com sangue no material causador do ferimento (13; $17,8 \%)$, ferimento superficial com agulha de sutura $(02 ; 2,7)$ e arranhões superficiais provocados por perfurocortantes sem sangue ou outros fluidos visíveis $(02 ; 2,7 \%)$.

Dentre as causas referidas de ocorrência dos acidentes, destacaram o descuido da equipe com o material contaminado $(16 ; 22,0 \%)$, o não uso de EP $(14 ; 19,2 \%)$, a viatura/veículo estar em movimento (14; 19,2\%), o espaço físico reduzido $(12 ; 16,4 \%)$, o local inadequado para a limpeza dos artigos

Tabela 1 - Caracterização dos profissionais, grupo saúde e não saúde, do atendimento pré-hospitalar. Goiânia-GO, 2007

\begin{tabular}{|c|c|c|c|c|c|c|c|}
\hline \multirow{2}{*}{ Variáveis } & \multirow{2}{*}{ Total } & \multicolumn{2}{|c|}{$\begin{array}{l}\text { Grupo saúde } \\
(\mathrm{N}=101)\end{array}$} & \multicolumn{2}{|c|}{$\begin{array}{l}\text { Grupo não saúde } \\
\quad(\mathrm{N}=76)\end{array}$} & \multirow{2}{*}{$c^{2}$} & \multirow{2}{*}{$p$} \\
\hline & & $\mathrm{N}$ & $(\%)$ & $\mathrm{N}$ & $(\%)$ & & \\
\hline \multicolumn{8}{|l|}{ Sexo } \\
\hline Masculino & 101 & 29 & $(28,7)$ & 72 & $(71,3)$ & 74,5 & 0,00 \\
\hline Feminino & 76 & 72 & $(94,7)$ & 4 & $(5,3)$ & & \\
\hline \multicolumn{8}{|c|}{ Faixa etária (anos) } \\
\hline$\leq 30$ & 102 & 56 & $(54,9)$ & 46 & $(45,1)$ & & \\
\hline$>30$ & 75 & 45 & $(60,0)$ & 30 & $(40,0)$ & 0,27 & 0,6 \\
\hline \multicolumn{8}{|l|}{ Carga horária } \\
\hline$\leq 44$ horas & 126 & 68 & $(54,0)$ & 58 & $(46,0)$ & & \\
\hline$>44$ horas & 51 & 33 & $(64,7)$ & 18 & $(35,3)$ & 1,3 & 0,25 \\
\hline \multicolumn{8}{|c|}{ Tempo de atuação } \\
\hline$\leq 5$ anos & 86 & 79 & $(91,9)$ & 7 & $(8,1)$ & & \\
\hline$>5$ anos & 91 & 22 & $(24,2)$ & 69 & $(75,8)$ & 79,9 & 0,00 \\
\hline
\end{tabular}


$(05 ; 6,8 \%)$, a ausência de recipiente para descarte de perfurocortante $(04 ; 5,5 \%)$, a falta de experiência $(04 ; 5,5 \%)$, recipientes para descarte de perfurocortante cheios $(02 ; 2,7 \%)$ e inadequados/improvisados $(02 ; 2,7 \%)$.

A maioria dos acidentes aconteceu durante a realização de procedimentos (47/73; 64,4\%). Durante a execução de procedimentos considerados invasivos (37/47, 78,7\%), como acesso vascular, aspiração de vias aéreas, administração de medicamentos, realização de sutura, de flebotomia, instalação de dreno torácico e intubação orotraqueal e durante aqueles considerados não invasivos (10/47; 21,3\%), como imobilização, remoção e exposição das vítimas.

Nas ocasiões pós-realização de procedimentos, os acidentes $(04 / 73 ; 5,5 \%)$ ocorreram durante a retirada do acesso vascular $(02 / 4 ; 50,0 \%)$ e o transporte das vítimas (02/4; 50,0\%). Também houve acidentes durante o manuseio de artigos, fora do momento do atendimento, propriamente dito (22/73; $30,1 \%)$, após o uso desses $(21 / 22 ; 95,4 \%)$ e durante o manejo de resíduos da assistência $(01 / 22 ; 4,6 \%)$.

Identificou-se que as medidas pós-exposição adotadas pela maioria dos sujeitos limitou-se aos cuidados locais com água e sabão (39/73; 53,4\%), enquanto que 46,5\% (34/73) acrescentaram a notificação do acidente e a investigação sorológica do paciente fonte.

Todos os sujeitos referiram inexistência de um serviço especializado de orientação e/ou encaminhamento dos acidentados e $12,9 \%$ (23/177) relataram nunca ter recebido orientações sobre medidas de biossegurança. As Tabelas 2 e 3 apresentam as variáveis de comportamento de risco dos sujeitos que podem influenciar na ocorrência e na gravidade dos acidentes, comparando as proporções e a significância estatística entre os grupos saúde e não saúde.

Realizou-se análise univariada das variáveis de comportamento de risco identificadas entre os profissionais do APH, segundo os grupos saúde e não saúde. Enquanto a totalidade do grupo saúde que referiu reencapar agulhas e não usar máscara, nem óculos protetores e nem uniforme completo sofreram acidente com $\mathrm{MB}$, a proporção daqueles que adotaram todas essas práticas e que referiram acidente com MB foi de 49,4\%, $35,4 \%, 43,2 \%, 56,7 \%$, respectivamente, e essa diferença foi estatisticamente significativa.

Enquanto $88,1 \%$ do grupo saúde que não descartaram os perfurocortantes apropriadamente referiram acidente, nenhum que descartou corretamente se acidentou $(p<0,05)$. No grupo não saúde, 18,4\% referiram acidentes, sendo que nenhum relatou uso de máscara, óculos protetores, uniforme completo e descarte adequado de perfurocortante. Em relação ao uso de luvas, $26,1 \%$ daqueles que não utilizaram sofreram acidentes $(p=0,067)$.

Dentre aqueles que referiram esquema completo (três doses) contra hepatite $B, 14,6 \%$ (19/130) afirmaram a realização do anti-HBs. O esquema incompleto (com uma ou duas doses) foi referido por 47 (61,8\%) indivíduos.

Verificou-se diferença nas proporções entre os vacinados e não-vacinados contra hepatite B (3,4\% e 27,7\%), sendo que os últimos apresentaram 10,7 (IC 1,3 - 86,9) vezes mais chances de terem sido acidentados quando comparados aos vacinados. Em relação à vacina contra tétano, embora a proporção de acidentes tenha sido maior entre os não-vacinados a diferença não foi estatisticamente significante.

Tabela 2 - Variáveis de comportamento de risco entre profissionais, grupo saúde e não saúde, do atendimento pré-hospitalar. Goiânia, GO. 2007

\begin{tabular}{|c|c|c|c|c|c|c|c|}
\hline \multirow{2}{*}{ Variáveis } & \multirow{2}{*}{ Total } & \multicolumn{2}{|c|}{$\begin{array}{l}\text { Grupo saúde } \\
(\mathrm{N}=101)\end{array}$} & \multicolumn{2}{|c|}{$\begin{array}{l}\text { Grupo não saúde } \\
\qquad(\mathrm{N}=76)\end{array}$} & \multirow{2}{*}{$C^{2}$} & \multirow[b]{2}{*}{$p$} \\
\hline & & $N$ & $(\%)$ & $\mathrm{N}$ & $(\%)$ & & \\
\hline \multicolumn{8}{|l|}{ Uso de luvas } \\
\hline Sim & 131 & 101 & $(77,1)$ & 30 & $(22,9)$ & & \\
\hline Não & 46 & 0 & $(0,0)$ & 46 & $(100,0)$ & 79,5 & 0,00 \\
\hline \multicolumn{8}{|c|}{ Uso de máscara } \\
\hline Sim & 65 & 65 & $(100,0)$ & 0 & $(0,0)$ & & \\
\hline Não & 112 & 36 & $(32,1)$ & 76 & $(67,9)$ & 74,5 & 0,00 \\
\hline \multicolumn{8}{|c|}{ Uso de óculos protetor } \\
\hline Sim & 74 & 74 & $(100,0)$ & 0 & $(0,0)$ & & \\
\hline Não & 103 & 27 & $(26,2)$ & 76 & $(73,8)$ & 92,7 & 0,00 \\
\hline \multicolumn{8}{|c|}{$\begin{array}{l}\text { Descarte apropriado de material perfuro- } \\
\text { cortante }\end{array}$} \\
\hline Faz & 34 & 34 & $(100,0)$ & 0 & $(0,0)$ & & \\
\hline Não Faz & 143 & 67 & $(46,8)$ & 76 & $(53,2)$ & 29,5 & 0,00 \\
\hline \multicolumn{8}{|c|}{ Reencapagem de agulha } \\
\hline Faz & 83 & 83 & $(100,0)$ & 0 & $(0,0)$ & & \\
\hline Não Faz & 94 & 18 & $(19,1)$ & 76 & $(80,9)$ & 114,3 & 0,00 \\
\hline \multicolumn{8}{|c|}{ Uniforme completo* } \\
\hline Sim & 97 & 97 & $(100,0)$ & 0 & $(0,0)$ & & \\
\hline Não & 80 & 4 & $(5,0)$ & 76 & $(95,0)$ & 157,6 & 0,00 \\
\hline
\end{tabular}

* O uso da calça comprida e jaqueta de mangas compridas com punho. 
Tabela 3 - Vacinação contra hepatite B e tétano, referida pelos profissionais, grupo saúde e não saúde, do atendimento pré-hospitalar. Goiânia, GO. 2007.

\begin{tabular}{|c|c|c|c|c|c|c|c|}
\hline \multirow[b]{2}{*}{ Imunização } & \multirow[b]{2}{*}{ Total } & \multicolumn{2}{|c|}{$\begin{array}{l}\text { Grupo saúde } \\
\qquad(\mathrm{N}=101)\end{array}$} & \multicolumn{2}{|c|}{$\begin{array}{l}\text { Grupo não saúde } \\
\qquad(\mathrm{N}=76)\end{array}$} & \multirow[b]{2}{*}{$c^{2}$} & \multirow[b]{2}{*}{$p$} \\
\hline & & $\mathrm{N}$ & $(\%)$ & $\mathrm{N}$ & $(\%)$ & & \\
\hline \multicolumn{8}{|l|}{ Vacinação contra hepatite B } \\
\hline Sim & 130 & 101 & $(77,7)$ & 29 & $(22,3)$ & & \\
\hline Não & 47 & 0 & $(0,0)$ & 47 & $(100,0)$ & 81,9 & 0,00 \\
\hline \multicolumn{8}{|l|}{ Vacinação contra tétano } \\
\hline Sim & 155 & 86 & $(55,5)$ & 69 & $(44,5)$ & & \\
\hline Não & 22 & 15 & $(68,2)$ & 7 & $(31,8)$ & 0,8 & 0,37 \\
\hline
\end{tabular}

\section{DISCUSSÃO}

O perfil dos profissionais do serviço de APH no município de Goiânia-GO assemelha-se ao de serviços de outras regiões do país ${ }^{(6,11-12)}$, quanto à predominância do sexo masculino e tempo de atuação entre 1 a 5 anos, a diferença restringe-se à idade dos profissionais ${ }^{(6,11-12)}$.

Conforme os dados de caracterização dos sujeitos e a ocorrência de acidentes, somente a variável tempo de atuação, > cinco anos foi associada a acidentes entre os profissionais do grupo saúde, enquanto no grupo não saúde, a variável carga horária > 44 horas mostrou essa associação. A idade, superior a 30 anos, mostrou-se como um fator de proteção para acidentes no grupo não saúde. Pode-se inferir, que o fator idade relaciona-se ao alcance de uma maturidade pessoal podendo influenciar na postura profissional. Já o tempo de atuação, associado à ocorrência de acidentes, pode interferir na adesão a medidas preventivas ${ }^{(3,13)}$.

O fato de a maioria dos sujeitos trabalhar mais de 44 horas semanais e possuir mais de um emprego, caracterizando uma sobrecarga e acúmulo de jornadas de trabalho, já foi identificado como fator de risco para a ocorrência de acidentes com $M B$ em serviços de urgência e emergência fixos ${ }^{(14)}$ e recentemente, investigado em um serviço de $\mathrm{APH}^{(5)}$, dado que não pode ser ignorado, principalmente, ao considerar as peculiaridades desses serviços.

A prevalência global de acidentes com MB encontrada $(41,2 \%)$ permanece na média, quando comparada a outras pesquisas com profissionais de serviços de APH, 19,8\% ${ }^{(9)}$; $20,6 \%{ }^{(3-4)} ; 27,3 \%^{(13)} ; 68,3 \%^{(5)}$ e $72,5 \%^{(12)}$. Esses índices mostram a necessidade de implementação de medidas preventivas específicas para esse grupo.

Os enfermeiros $(28,7 \%)$, os médicos $(26,0 \%)$ e técnicos em enfermagem $(26,0 \%)$ foram os profissionais que mais se acidentaram com MB corroborando dados da literatura nacional e internacional sobre serviços de $\mathrm{APH}^{(3-9)}$. A alta prevalência de acidentes com esses profissionais em serviços de $\mathrm{APH}$, pode estar relacionada à maior exposição durante um atendimento, à realização de procedimentos invasivos, à submissão a grande carga de estresse e à cobrança por resultados rápidos e eficazes ${ }^{(4-5,13,15)}$.

Entretanto merece destaque os acidentes com MB envolvendo o grupo não saúde $(18,4 \%)$. Estudos que analisaram estes acidentes entre a equipe multiprofissional do $\mathrm{APH}$, identificaram, entre profissionais do referido grupo (não saúde) índices de acidentes que variavam de $7,3 \%{ }^{(5)}, 16,7 \%{ }^{(4)}$ até $24,0 \%{ }^{(9)}$. Dados que precisam ser considerados nas políticas de segurança para os trabalhadores de APH.

Do total de acidentes, categorizados como graves $(n=45)$ e não graves $(n=28)$, a maioria $(86,3 \%)$ envolveu sangue, o mesmo foi identificado em estudos tanto entre profissionais de Estabelecimentos Assistenciais de Saúde ${ }^{(16)}$ quanto em serviços de $\mathrm{APH}^{(6,15)}$

O "descuido da equipe com o material contaminado" foi a causa mais referida para a ocorrência dos acidentes envolvendo $\mathrm{MB}$. Esse tema tem importância abrangente ao considerar que o não cuidado com o resíduo contaminado pode ter conseqüências tanto para o profissional que o gerou como para o restante da equipe. Assim vale recorrer à legislação que responsabiliza o profissional que gerou o resíduo pelo seu descarte ${ }^{(17)}$. Porém, no contexto dos serviços de APH, não é possível afirmar que o descuido seja de responsabilidade direta apenas do profissional do $\mathrm{APH}$, pois se relacionam a essa condição situações referidas pelos próprios trabalhadores, como o não uso de equipamentos de proteção $(19,2 \%)$, a ausência $(5,5 \%)$ ou a superlotação $(5,4 \%)$ de recipiente para descarte de perfurocortante ou sua utilização inadequada $(5,4 \%)$, que somadas representaria a maioria das causas dos acidentes neste estudo.

Esses dados levam ao questionamento da relação do "descuido" com o trabalho no APH. Será que o fato de configurar-se como serviço de emergência levaria a essa postura por parte dos profissionais, no sentido de priorizar o atendimento e transporte do paciente? Estudos complementares se fazem necessários para aprofundamento nessa questão. Entretanto, ressalta-se que esses serviços precisam, também, oferecer segurança para todos os seus profissionais, fato que vai além da responsabilidade individual.

Outras causas referidas pelos próprios profissionais para acidentes com MB estão relacionadas ao material perfurocortante (10,9\%), causador de 32 (43,8\%) acidentes. Essa temática precisa receber intervenções imediatas e pautadas na legislação vigente ${ }^{(17)}$, considerando que $46,8 \%$ dos sujeitos afirmaram não descartar, frequentemente, de modo apropriado esses resíduos, denotando um risco individual e coletivo. Além do mais, essa variável apresentou associação com 
a ocorrência de acidentes envolvendo MB. Acredita-se que a implantação de dispositivos de segurança, obrigatória no país ${ }^{(18)}$, pode contribuir para reduzir os acidentes com perfurocortantes, como já verificado nos Estados Unidos ${ }^{(5)}$.

Taxas de $28,6 \%{ }^{(6)}, 39,1 \%{ }^{(9)}, 40,8 \%{ }^{(4)}, 72,5 \%^{(12)}$ de acidentes envolvendo materiais perfurocortantes entre profissionais do APH já foram encontradas. Entretanto, estes índices se assemelham aos encontrados entre profissionais da área da saúde de serviços de urgência e emergência fixos. O que chama a atenção é a ocorrência de acidentes entre profissionais, ditos do grupo não saúde que, teoricamente, não se envolvem com materiais perfurocortantes durante suas atividades laborais e isso instiga reflexões e questionamentos. Estudo mostrou que, dentre $16,7 \%$ dos acidentes entre profissionais do grupo não saúde (condutores), 72,7\% envolveram perfurocortantes ${ }^{(4)}$; e outro, onde a taxa de acidentes entre condutores foi de $24,0 \%, 66,7 \%$ envolveram perfurocortantes ${ }^{(9)}$. Evidências apontam que este fato acontece ${ }^{(4)}$; porém, as pesquisas parecem negligenciar esta discussão.

Outro risco no manuseio dos resíduos é o hábito de reencapar agulhas, relatado por todos os profissionais do grupo saúde, demonstrando que essa conduta de risco, proibida pela legislação ${ }^{(17)}$ faz parte da rotina de profissionais tanto em $\mathrm{EAS}^{(16)}$ e em serviços de $\mathrm{APH}^{(8,13)}$.

A adesão aos equipamentos de proteção foi baixa, como em outros estudos ${ }^{(3,15,19)}$ com a mesma população. Fato intrigante é que os sujeitos identificaram essa prática como causa para de acidentes com MB.

Verificou-se que o grupo não saúde tem se exposto a fluidos orgânicos nas atividades laborais no APH e apresentaram baixa adesão aos EP, aumentando o risco de acidentes com $\mathrm{MB}$, embora a adesão aos EP do grupo saúde ainda esteja abaixo do esperado. Esses dados sinalizam para a necessidade do estabelecimento de uma política de medidas preventivas para ambos os grupos no que diz respeito a esclarecimentos e treinamentos relacionados à biossegurança para o RB, pois ambos estão expostos ${ }^{(4,19)}$. Destaca-se que o uso de diferentes estratégias de prevenção de acidentes neste grupo ainda não foi investigado.

Pesquisa com o objetivo de avaliar a adesão às PP verificou que profissionais do APH não alcançaram adequação das atitudes e conhecimentos para uso de máscara, óculos e os demais EP, e os condutores relataram atitude inadequada para todos os itens ${ }^{(3)}$. Outro estudo, realizado com enfermeiros do $\mathrm{APH}$, mostrou que, apesar de perceberem a importância do uso dos EP e terem conhecimento da sua exposição ao RB, houve negligência no uso de máscara e óculos de proteção nos atendimentos; os autores concluíram que a percepção não foi suficiente para o emprego dos EP na prática ${ }^{(15)}$.

Além da "não adesão aos EP", os aspectos "viatura em movimento", não encontrado em outras pesquisas que envolvem $\mathrm{APH}$, "espaço físico reduzido" e "falta de local adequado para a limpeza de materiais" foram apontados pelos profissionais como fatores causais de acidentes com MB. Há que se considerar que esses fatores dificultam a realização de procedimentos e potencializam o risco de ocorrência de acidentes, particularidades que devem ser consideradas ao analisar os comportamentos dos profissionais e planejar ações, em conjunto, de educação permanente, as quais deveriam contemplar também a "inexperiência no trabalho de $\mathrm{APH}^{\text {" (5,5\%) }}$ referida como causa de acidentes envolvendo $\mathrm{MB}$.

Uma medida de proteção, individual e coletiva, implícita nas PP é a imunização dos profissionais ${ }^{(10)}$, que foi referida pela maioria dos sujeitos, tanto para hepatite $\mathrm{B}(73,4 \%)$ como para tétano $(87,6 \%)$. Destaca-se que para a hepatite B o índice foi maior que o encontrada em estudo na mesma região com profissionais do APH $(38,6 \%)^{(13)}$. Em contrapartida, índices superiores já foram descritos na literatura com a mesma população $69,1 \%{ }^{(19)}$.

O conjunto de medidas pós-exposição recomendado ${ }^{(20)}$ foi negligenciado pela maioria daqueles que relataram acidentes com MB. Sabe-se que no caso deste tipo de acidente, a imunização aliada a condutas pós-exposição, incluindo notificação dos casos, atua como fatores protetores à saúde do trabalhador.

A normativa brasileira ${ }^{(20)}$ que trata das orientações em caso de exposição a MB considera como trabalhadores da área de saúde todos os profissionais do setor saúde que atuam, direta ou indiretamente, em atividades em que há risco de exposição a sangue e a outros $\mathrm{MB}$ o que inclui os integrantes do $\mathrm{APH}$.

Os índices de notificação de acidentes com profissionais do $\mathrm{APH}$ ainda são pouco conhecidos, mas aqueles já identificados mostram o extremo descaso com essa conduta, 18,4\% ${ }^{(4)}$, $8,7 \%{ }^{(9)}$ até mesmo, nulo ${ }^{(13)}$. A subnotificação identificada no presente estudo $(53,4 \%)$ pode estar relacionada à falta de um serviço especializado para o atendimento às vítimas de acidentes e notificação dos casos e à falta de informação desses trabaIhadores, dificultando a tomada das condutas pós-exposição, de onde se pode inferir maior vulnerabilidade desse grupo.

\section{CONCLUSÃO}

Verificou-se alta prevalência $(44,2 \% ; 73 / 177)$ de acidentes envolvendo $\mathrm{MB}$ entre os profissionais do $\mathrm{APH}$, apesar de ter sido maior no grupo saúde (58,4\%), um índice de 18,4\% de acidentes entre o grupo, dito como não saúde merece grande atenção. Este achado pode revelar uma nova tendência e necessidade de enfoque de investigações na área de RB em interface com os serviços de APH.

Os acidentes caracterizados como graves foram os mais expressivos assim como aqueles envolvendo sangue. A maioria aconteceu durante a realização de um procedimento e cuja causa principal relatada foi o descuido com material contaminado.

Medidas protetoras frente ao RB foram negligenciadas por ambos os grupos, como a adesão aos EP, o descarte adequado de perfurocortantes e a vacinação completa contra hepatite B e a realização do anti-HBs. Medidas pós-exposição foram parcialmente aplicadas na maioria dos casos.

As variáveis de comportamento de risco para ocorrência de acidentes como o não uso de luvas, máscara e óculos protetor, o descarte inapropriado de material perfurocortante, o uso da vestimenta incompleta, o re-encape de agulhas e a não imunização contra hepatite $B$ foram estatisticamente significativas 
$(p<0,05)$ para a ocorrência de acidente com MB para ambos os grupos.

Os dados evidenciaram a importância da adesão às medidas preventivas por todos os profissionais - grupo saúde e não saúde - e a necessidade de estruturação e implementação de um sistema de vigilância e controle dos acidentes, envolvendo $M B$, voltado para o grupo específico de profissionais do APH. Essas ações possibilitarão conhecer as especificidades dos acidentes com esse grupo e o acompanhamento do profissional, além de fornecer subsídios para programas de educação permanentes para essa população.

Devem ser realizados novos estudos direcionados ao serviço e aos profissionais, de forma conjunta - grupo saúde e não saúde - que atuam em APH, com vistas a elaborar, aplicar e avaliar estratégias preventivas que atendam suas especificidades.

\section{REFERÊNCIAS}

1. Ministério da Saúde. Secretaria Estadual de Saúde de Goiás. Serviço de Atendimento Móvel de Urgência. Goiás: Secretaria Estadual da Saúde; 2011.

2. Ministério da Saúde (Brasil). Portaria nº. 2048, de 5 de novembro de 2002. Regulamenta o atendimento das urgências e emergências. Diário Oficial da União 12 nov 2002;Seção 1.

3. Lopes AC, Oliveira AC, Silva JT, Paiva MH. Adherence to standard precautions by the public pre-hospital health team in Belo Horizonte, Minas Gerais State, Brazil. Cad Saúde Pública 2008;24(6):1387-96.

4. Oliveira AC, Lopes ACS, Paiva MHRS. Occupational accidents due to exposure to biological material in the multidisciplinary team of the emergency service. Rev Esc Enferm USP 2009;43(3):677-83.

5. Soerensen AA, Moriya TM, Hayashida M, Robazzi MLCC. Accidents with biological material among health workers providing mobile pre-hospital care. Rev Enferm UERJ 2009;17(2):234-9.

6. Vegian CFL, Monteiro MI. Living and working conditions of the professionals of the a Mobile Emergency Service. Rev Latino-Am Enferm 2011;19(4):1018-24.

7. Boal WL, Leiss JK, Ratcliffe JM, Sousa S, Lyden JT, Li J, et al. The national study to prevent blood exposure in paramedics: rates of exposure to blood. Int Arch Occup Environ Health 2010;83:191-9.

8. Harris SA, Nicolai LA. Occupational exposures in emergency medical service providers and knowledge of and compliance with universal precautions. Am J Infect Control 2010;38(2):86-94.

9. Paiva MHRS, Oliveira AC. Determinant factors and conduct in post-accident with biological material among pre-hospital professionals. Rev Bras Enferm 2011;64(2):268-73.

10. Siegel JD, Rhinehart E, Jackson M, Chiarello L. Health care infection control practices advisory committee. Guideline for isolation precautions: preventing transmission of infectious agents in health care settings. Am J Infect Control 2007;35(10 Suppl 2):S65-4.

11. Silva JA, Paula VS, Almeida AJ, Villar LM. Investigation of biological accidents among health care workers. Esc Anna Nery Rev Enferm 2009;13(3):508-16.

12. Zapparoli A, Marziale MHP. Occupational risk in basic and advanced emergency life support units. Rev Bras Enferm 2006;59(1):41-6.

13. Florêncio VB, Rodrigues CA, Pereira MS, Souza ACS. Adesão às precauções padrão entre os profissionais da equipe de resgate pré-hospitalar do corpo de bombeiros de Goiás. Rev Eletr Enferm. [periódico na internet]. 2003 [acesso em 22 jan 2012];5(1):43-8. Disponível em: http://www. revistas.ufg.br/index.php/fen/article/viewArticle/770

14. O'Dwyer G, Matta IEA, Pepe VLE. Evaluation of emergency hospital services in the state of Rio de Janeiro. Ciênc Saúde Coletiva 2008;13(5):1637-48.

15. Mafra DAL, Santana JCB, Fonseca IC, Silva MP, Viana JX. Perception of Nurses on the importance of the use of Individual Protection Equipment for Biological Risks in a Service of Mobile Urgency Care. Mundo Saúde 2008;32(1):31-8.

16. Gomes AC, Agy LL, Malaguti SE, Canini SRMS, Cruz EDA, Gir E. Occupational Accidents with biological material and the nursing team in a teaching hospital. Rev Enferm UERJ 2009;17(2):220-3.

17. Ministério do Trabalho e Emprego (Brasil). Portaria $n^{\circ}$. 485, de 11 de novembro de 2005. Aprova a norma regulamentadora $\mathrm{n}^{\circ} .32$ sobre segurança e saúde no trabalho em estabelecimentos de saúde. Diário Oficial da União 16 nov 2005;Seção 1.

18. Ministério do Trabalho e Emprego (Brasil). Portaria $\mathrm{n}^{\circ}$ .1.748, de 30 de agosto de 2011. Institui o plano de Prevenção de Riscos de Acidentes com Materiais Perfurocortantes e altera a Norma Regulamentadora $n^{\circ}$. 32, que trata da segurança e saúde no trabalho em estabelecimentos de saúde. Diário Oficial da União 31 out 2011;Seção 1.

19. Lima CCCM, Anazário IS, Oliveira MVM, Silva SC, Freire SC, Ricarte MC, et al. Biosafety in a prehospitalization care setting. Rev Inst Ciênc Saúde 2007;25(1):15-22.

20. Ministério da Saúde. Recomendações para atendimento e acompanhamento de exposição ocupacional a material Biológico: HIV e Hepatites B e C. Brasília, DF: O Ministério; 2010. 